\title{
Estudo do veneno de Tityus serrulatus (Scorpiones; Buthidae) procedente do estado da Bahia, Brasil
}

\author{
Tiago Ferreira da Silva ${ }^{1}$ \\ Luciana Lyra Casais-e-Silva \\ Aryon de Almeida Barbosa-Júnior ${ }^{3}$ \\ Rejâne Maria Lira-da-Silva ${ }^{4}$
}

\begin{abstract}
Resumo
O escorpiāo Tityus serrulatus é conhecido como a espécie mais importante, do ponto de vista médico, pois é o que causa os acidentes mais graves registrados (para) no território brasileiro. Este trabalho trata da caracterização do veneno de escorpiōes T. serrulatus, procedentes de diferentes regiões da Bahia-Brasil, através da obtenção da $\mathrm{DL}_{50}$, a observação da existência do edema pulmonar e caracterização dos distúrbios neurológicos induzido pela peçonha. O veneno foi obtido através de estímulo elétrico, utilizando-se de um extrator desenvolvido especificamente para esse fim. Camundongos Swiss machos (18-22g) foram utilizados para avaliar a toxicidade pelo método de Finney (1971). A indução de edema pulmonar foi calculada pela diferença de peso úmido dos pulmôes controle e teste. $\mathrm{A} \mathrm{DL}_{50}$ obtida foi de $96,3 \mu \mathrm{g} /$ camundongo, considerada alta, demonstrando a baixa toxicidade do veneno. A toxicidade observada foi de 3 a 7 vezes menor que a do veneno da mesma espécie em outras regiōes do Brasil (São Paulo e Minas Gerais). Nossos resultados demonstraram que o veneno de T. serrulatus do Estado da Bahia não induz o edema pulmonar agudo, tanto na avaliação do índice peso pulmonar/peso corporal quanto na avaliação morfológica. Esses fatores podem explicar a ausência de óbitos nos acidentes por Tityus serrulatus na Região Metropolitana do Salvador (RMS) e é concordante com dados de outros autores que, no período de 1982-2000, não registraram óbito nos acidentes escorpiônicos na mesma região.
\end{abstract}

Palavras-chave: escorpião - veneno; Tityus; T. serrulatus.

\section{INTRODUÇÃO}

Embora os envenenamentos por animais peçonhentos, incluindo-se escorpiōes, tenham atingido os homens desde a Antigüidade, apenas recentemente temos estudado a química natural e efeitos fisiológicos das toxinas presentes nos venenos. Historicamente, o interesse nos venenos dos escorpiōes aumentou graças ao perigo que representam para a saúde humana. (BROWNELL; POLIS, 2001)

Para Lourenço e Eickstedt (2003), são conhecidas hoje 1500 espécies, distribuídas em 20 famílias e 165 gêneros; desse total, apenas

\footnotetext{
${ }^{1}$ Bolsista de Iniciação Científica (FAPESB) de Ciências Biológicas da Universidade Federal da Bahia. Salvador - BA

2 Professora Adjunto. Escola Bahiana de Medicina e Saúde Pública; Faculdade Jorge Amado; União Metropolitana de Educação e Cultura (UNIME). Salvador - BA

${ }^{3}$ Pesquisador Titular. Laboratório de Histopatologia do Centro de Pesquisas Gonçalo Muniz - FIOCRUZ. Salvador - BA

${ }^{4}$ Professora Adjunto do Departamento de Zoologia do Instituto de Biologia (UFBA). Salvador - BA

Correspondência para / Correspondence to:

Rejâne Maria Lira-da-Silva

Instituto de Biologia da UFBA - Núcleo Regional de Ofiologia e Animais Peçonhentos da Bahia.

Rua Barão de Geremoabo, s/n - Campus de Ondina.

40170-290. Salvador - Bahia - Brasil.

Tel.: (71)3263-6564; Fax: (71)3263-6511.

E-mail: rejane@ufba.br.
}

R. Ci. méd. biol., Salvador, v. 4, n. 1, p. 24-31, jan./abr. 2005 
25 espécies são consideradas de importância médica, o que representa $2 \%$ da fauna escorpiônica mundial. No Brasil, a escorpiofauna é representada pelas famílias Ischnuridae, Chactidae, Bothriuridae e Buthidae, apresentando 15 gêneros e 86 espécies, destacando-se essa última com as principais espécies de importância médica: Tityus serrulatus, T. bahiensis, T. trivittatus e T. stigmurus. (MANUAL..., 1999)

Todos os escorpiōes de interesse médico, no Brasil, estão agrupados no gênero Tityus e, dentre eles, as três espécies mais importantes são T. serrulatus, T. bahiensis e T. stigmurus (LIRADA-SILVA; AMORIM; BRAZIL, 2000). O Tityus serrulatus é a espécie de escorpião mais importante, do ponto de vista médico, pois é o que causa maior número de acidentes e que gera os piores envenenamentos (MANUAL..., 1999). A Bahia possui $25 \%$ das espécies descritas ou mencionadas para o Brasil, 5 gêneros com 19 espécies no total, representadas, na sua maioria, pelas famílias Bothriuridae e Buthidae. (FUKUTANI et al., 2004)

A espécie Tityus serrulatus mede entre $6 \mathrm{e}$ $7 \mathrm{~cm}$, possui uma coloração marrom-escuro e pedipalpos e patas amarelados. A cauda, que também é amarelada, apresenta uma serrilha dorsal nos dois últimos segmentos e uma mancha escura no lado ventral da vesícula (MANUAL..., 1999). É um animal (endêmico) freqüente no Brasil e ocorre do estado da Bahia, Espírito Santo, Minas Gerais até o Rio de Janeiro, São Paulo, Paraná e Goiás, abrangendo as regiōes Nordeste, Centro-Oeste e Sudeste (MANUAL..., 1999). Originalmente, vivia em ambientes de mata de transição, florestas secas, cerrados e caatinga (LOURENÇO; CLOUDSLEYTHOMPSON, 1999). Atualmente, essa espécie tem habitado locais com um mínimo de vegetação e se proliferado amplamente em cidades, por ser comprovadamente partenogenética e ecologicamente "oportunista", ou seja, adapta-se facilmente a novas condições do ambiente. Esses podem ser os motivos do alto índice de acidentes registrado em nosso país. (LOURENÇO et al., 1996)

O estudo sobre o veneno de escorpiōes e o respectivo processo de envenenamento, no
Brasil, começou no início do século por Maurano (1915) e Vital Brazil (1918), seguido de Magalhães e Tupinambá (1938), todos na região Sudeste. Rosenfeld (1976) acreditava ser a toxina escorpiônica neurotrópica e neurotóxica, devido à observação de que, em inúmeros casos humanos, ela provocava manifestaçôes clínicas graves, principalmente em crianças. Impressionado com esses sintomas, realizou grande número de experiências em animais, demonstrando a ação do veneno sobre os núcleos vagais e postulando que o processo de intoxicação deveria se caracterizar por lesões bulbares. Schvartsman (1992), seguindo mesma linha de investigação, verificou, em trabalhos experimentais, que as lesões provocadas pelo envenenamento escorpiônico situavam-se preferencialmente no bulbo, mesencéfalo e protuberância, o que explicaria as alterações observadas nos sistemas digestivo, circulatório, respiratório, urinário e de controle de temperatura. No entanto, estudos posteriores demonstraram que o local de ação da peçonha não deveria estar localizado no sistema nervoso central, visto que a maioria dos seus efeitos ainda era observada em animais espinalectomizados e em órgãos isolados (HERING et al., 1992).

Hoje, está bem estabelecido que a toxina escorpiônica age em sítios específicos dos canais de sódio, produzindo tanto despolarização como bloqueio das células excitáveis, a depender da toxina presente (HERING; AZEVEDO-MARQUES; CUPO, 1992). Em decorrência disso, pode ocorrer liberação maciça de catecolaminas e acetilcolina pelas terminaçôes nervosas pósganglionares dos sistemas simpático e parassimpático e na medula da adrenal, as quais, atuando nos diferentes setores do organismo, são responsáveis pela maioria dos sinais e sintomas observados no envenenamento escorpiônico humano, podendo estas evoluir para manifestações gastrointestinais, respiratórias, cardiovasculares e neurológicas. (CUPO; AZEVEDO-MARQUES; HERING, 2003)

$\mathrm{Na}$ Bahia, esta espécie foi responsável por 28,8\% dos acidentes escorpiônicos, no período de 1982-2000 e, dentre esses, $75 \%$ foram considerados moderados ou graves (LIRA-DA-SILVA et al., 2001), reforçando a sua importância 
médica também aqui em nosso Estado. $\mathrm{Na}$ cidade do Salvador, para o mesmo período, $T$. serrulatus foi responsável por uma percentagem menor de acidentes $(13,4 \%)$ e de maior gravidade $(10,8 \%)$ (LIRA-DA-SILVA et al., 2003).

O objetivo deste trabalho é caracterizar o veneno do escorpião T. serrulatus, (FIGURA 1) procedente da Região Metropolitana de Salvador, Bahia, Brasil, quanto à sua toxicidade, produção de efeitos neurotóxicos e edema pulmonar.

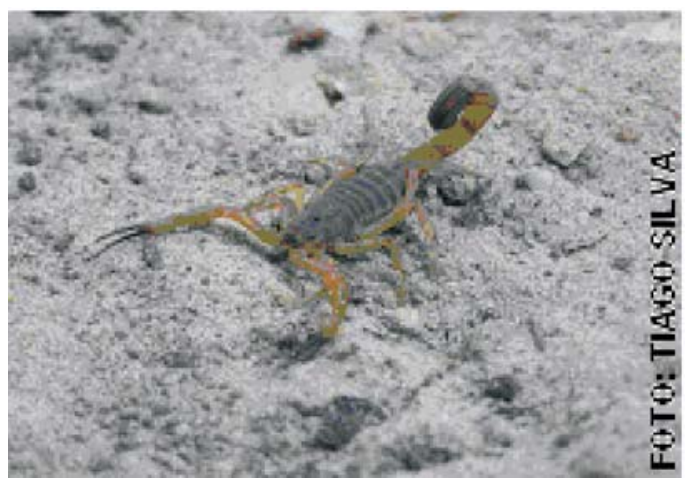

Figura 1 - Tityus serrulatus, Lutz e Mello (1922)

\section{MATERIAL E MÉTODOS}

O veneno de T. serrulatus foi obtido através de estimulação elétrica, segundo o método de Barrio e Vital Brazil (1949), de animais mantidos em cativeiro no Núcleo Regional de Ofiologia e Animais Peçonhentos da Bahia, Departamento de Zoologia, Instituto de Biologia, UFBA.

Utilizou-se de um extrator de veneno (FIGURA 2) desenvolvido especificamente para esse fim. O metassoma do animal foi imobilizado e o seu telson estimulado com choques de $10 \mathrm{~V}$, em intervalos médios de 5 segundos. $\mathrm{O}$ veneno liberado foi coletado em um vidro de relógio. Após a coleta, o veneno foi seco a vácuo, raspado com uma espátula, pesado em uma balança analítica (Modelo AND/ HR 200) e armazenado a uma temperatura de $-20^{\circ} \mathrm{C}$.

Para as extrações, realizadas em intervalos de três semanas, foram utilizados 22 animais coletados e (ou) doados, procedentes da Região

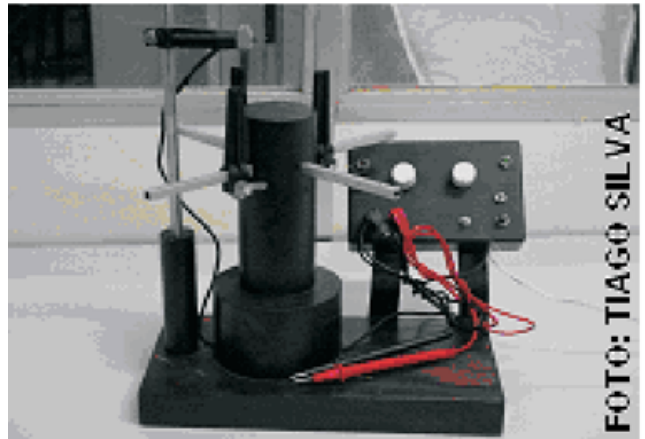

Figura 2 - Extrator de veneno desenvolvido no Núcleo Regional de Ofiologia e Animais Peçonhentos da Bahia.

Metropolitana do Salvador (RMS) e Centro-Sul Baiano, do estado da Bahia (QUADRO 1).

Foram utilizados camundongos albinos machos (Mus musculus), cedidos pelo Biotério da Escola de Veterinária da Universidade Federal da Bahia, que pesavam entre 18 e 22g, para os testes de toxicidade, e ratos Wister macho (Rattus rattus), cedidos pelo Centro de Pesquisa Gonçalo Muniz de Salvador (FIOCRUZ/BA), com peso entre 200 e $220 \mathrm{~g}$, para os testes de edema pulmonar e atividade neurotóxica. Os animais foram mantidos com água e ração $a d$ libitum, segundo as normas de utilização de animais em laboratório.

A toxicidade do veneno de T. serrulatus foi avaliada segundo o método de Finney (1971), pela determinação da dose letal 50\% $\left(\mathrm{DL}_{50}\right)$, em camundongos (via intraperitonial, i.p.), usando-se o volume de $0,5 \mathrm{ml}$ de uma solução de veneno, em solução salina $0,85 \%$ (w/ v) (as diferentes soluções foram obtidas através de sucessivas diluiçôes com intervalos de 1,5 a partir da dose inicial). A taxa de sobrevivência dos animais foi observada 24 e 48 horas após a inoculação do veneno.

A avaliação do edema pulmonar induzido pelo veneno de T. serrulatus foi feita pela determinação da diferença entre o peso dos pulmões de ratos injetados com veneno em relação ao dos de animais-controle, injetados com solução salina $0,85 \%$. Para tanto, grupos de animais foram injetados com veneno de T. serrulatus $(0,4 \mathrm{ou} 0,8 \mathrm{mg} / \mathrm{kg}$, i.v. $)$; o grupo-controle recebeu solução salina em volume equivalente. Após uma hora, os animais foram anestesiados com 


\begin{tabular}{|c|c|c|}
\hline RG/NOAP & ÁREA & PROCEDÊNCIA \\
\hline & REGIÃO METROPOLITANA DE SALVADOR & \\
\hline 6259 & & Camaçari/ Bahia \\
\hline 6125 & & Camaçari/ Bahia \\
\hline 6723 & & Itaparica/ Bahia \\
\hline 6736 & & Mata de São João/ Bahia \\
\hline 6737 & & Mata de São João/ Bahia \\
\hline 6738 & & Mata de São João/ Bahia \\
\hline 6740 & & Mata de São João/ Bahia \\
\hline 6742 & & Mata de São João/ Bahia \\
\hline 6189 & & Monte Gordo/ Camaçari/ Bahia \\
\hline 6193 & & Monte Gordo/ Camaçari/ Bahia \\
\hline 6195 & & Monte Gordo/ Camaçari/ Bahia \\
\hline 6256 & & Monte Gordo/ Camaçari/ Bahia \\
\hline 6809 & & Santo Amaro da Purificação/ Bahia \\
\hline 6810 & & Santo Amaro da Purificação/ Bahia \\
\hline \multicolumn{3}{|c|}{ CENTRO-SUL BAIANO } \\
\hline 6753 & & Maracás/Bahia \\
\hline 6754 & & Maracás/ Bahia \\
\hline 6755 & & Maracás/Bahia \\
\hline 6756 & & Maracás/ Bahia \\
\hline TOTAL & & 18 exemplares \\
\hline
\end{tabular}

Quadro 1: Procedências dos escorpiōes do estado da Bahia e seus respectivos registros (RG) no Núcleo de Ofiologia e Animais Peçonhentos da Bahia (NOAP), Instituto de Biologia UFBA.

éter etílico e sacrificados pela secção dos vasos cervicais, para a retirada dos pulmões, que foram imediatamente pesados em uma balança semi-analítica (HELMAC/ Modelo HM 1000), e o índice de edema determinado segundo a fórmula:

$$
\text { Índice }=\frac{\text { Peso do Pulmão }}{\text { Peso do Corpo }} \times 100
$$

Para a análise histológica, os pulmōes foram fixados em formol a $10 \%$, desidratados "overnight", em série crescente de 07 baterias de álcool (01 hora em cada bateria), diafanizados em 02 baterias de xilol e embebidos em 02 baterias de parafina, utilizando-se o Automatic Tissue Processor/Histokinette 2000Oे, no Laboratório de Histopatologia do Centro de Pesquisa Gonçalo Moniz (FIOCRUZ/Bahia).

Para a determinação da neurotoxicidade do veneno de T. serrulatus, foi feita a observação dos distúrbios neurológicos durante o período de uma hora após a injeção de 0,4 ou $0,8 \mathrm{mg} /$ $\mathrm{kg}$, i.v. (intravenosa) da peçonha. As alterações comportamentais, a condição respiratória, a salivação, a agitação e a letargia do grupo experimental foram comparadas com as observadas nos animais-controle, injetados com solução salina.

Os resultados foram expressos como médias \pm erro padrão da média (e.p.m.). As análises estatísticas foram feitas com o auxílio do programa Graphpad InStat, utilizando-se de testes de variância (ANOVA) com um nível de significância de $p<0,05$.

\section{RESULTADOS E DISCUSSÃO}

O veneno escorpiônico é secretado por um par de glândulas localizadas no último segmen- 
to caudal, o telson (BÜCHERL, 1978). Segundo Cupo, Azevedo-Marques e Hering (2003), esse veneno compreende uma mistura complexa de proteínas básicas de baixo peso molecular, associada a pequenas quantidades de aminoácidos e sais, sem atividade hemolítica, proteolítica, colinesterástica, fosfolipásica e sem atividade sobre o fibrinogênio. Esse veneno pode ser extraído utilizando-se desde a maceração do telson do animal em solução salina ou estímulo manual do prossoma do escorpião, até o método de estimulação elétrica (BÜCHERL; BUCKLEY, 1971). Utilizando a técnica de estimulação elétrica obtivemos (uma estimativa de) $252 \quad 29,85 \mu \mathrm{g}$ de veneno de Tityus serrulatus, quantidade inferior à obtida por Bücherl e Buckley (1971), para T. serrulatus $(697 \mu \mathrm{g})$ e para o T. bahiensis $(309 \mu \mathrm{g})$ do Sudeste do Brasil. Comparativamente, Bücherl (1978) obteve para T. serrulatus $75 \mu \mathrm{g}$ e 113 $\mu \mathrm{g}$ para o $\mathrm{T}$. bahiensis da mesma região; de um modo geral, essa variabilidade pode estar relacionada ao tamanho do escorpião, o regime de alimentação e frequiência de extrações. Depois de dez a quinze extrações, os animais tendem a diminuir a quantidade de veneno em $0,03 \mathrm{mg}$ por espécime (BÜCHERL; BUCKLEY, 1971). Segundo Bücherl e Buckley (1971), e Bücherl (1978), o máximo de veneno que um escorpião do gênero Tityus pode injetar em uma vítima é de 2 a $3 \mathrm{mg}$.

A peçonha dos espécimes estudados mostrou-se 7,7 vezes menos tóxica que o observado em diversos outros estudos que utilizaram a mesma espécie, porém provenientes de outras regiōes do Brasil, principalmente Regiáo Sudeste (BÜCHERL, 1978; KALAPOTHAKIS; CHAVEZ-OLORTEGUI, 1997; VIEIRA et al., 1996; NISHIKAWA et al., 1994).

A patogênese do edema pulmonar induzido pelo veneno escorpiônico é muito complexa. Dados de Freire-Maia, Campos e Amaral (1994) e de Amaral, Resende e Freire-Maia (1993) sugerem que essa ação decorre da ativação de mecanismos cardiogênicos e não cardiogênicos. Dentre os fatores cardiogênicos, incluem-se hipertensão arterial, provocada pelo veneno, que induz a falência do ventrículo esquerdo, aumento do retorno venoso e insufici- ência do miocárdio. Os mecanismos não cardiogênicos estariam relacionados com a liberação de substâncias vasoativas, que podem levar ao aumento da permeabilidade vascular (FREIRE-MAIA et al., 1970, 1976, 1994; MATTOS et al., 1997). Nossos resultados demonstraram que o veneno de $T$. serrulatus das regiôes estudadas não induz edema pulmonar, quando avaliado através da determinação do índice peso pulmonar/peso corporal e através da analise histológica. Diversos autores apontaram como a principal manifestação responsável pelo óbito em crianças com idades entre 3 e 9 anos, no Sudeste do Brasil (RAHAV; WEISS,1990; FREIRE-MAIA; CAMPOS; AMARAL, 1994; AMARAL; RESENDE; FREIRE-MAIA, 1993). De fato, não foi registrado, no envenenamento humano de $T$. serrulatus na Região Metropolitana do Salvador, a presença de edema pulmonar agudo (LIRA-DA-SILVA et al., 2001) no período de 1982-2000. A ausência de atividade edematogênica no pulmão, aliada à baixa toxicidade, justificam a ausência de óbitos nos acidentes escorpiônicos dessa região (LIRA-DASILVA et al., 2001). Esses dados sugerem uma variação regional na composição do veneno, o que estaria refletindo uma diferença nas características clínicas do envenenamento.

A atividade neurotóxica encontrada no presente trabalho foi caracterizada pela presença de hiperatividade, taquipnéia, letargia, congestão ocular, piloereção e sialorréia. Esses dados são semelhantes aos obtidos por Magalhães e colaboradores (1998), que também observaram distúrbios neurológicos caracterizados pela liberação de catecolaminas excessivas, utilizando a toxina isolada do veneno de $T$. serrulatus, tityustoxina (TsTx), que imita muitos efeitos dos estímulos elétricos, como o aumento da liberação de glutamato e acetilcolina (FERNANDES et al., 2004). Sintomas semelhantes também foram relatados em envenenamentos humanos por diversos autores na regiāo Nordeste (LIRA-DA-SILVA et al., 2000, 2001) e na região Sudeste (HERING; AZEVEDOMARQUES; CUPO, 1992; FREIRE-MAIA; CAMPOS; AMARAL, 1994; CUPO et al.,1994; MAGALHÃES et al., 1998). 


\section{CONCLUSÕES}

Podemos concluir que espécimes de $T$. serrulatus de nosso Estado produzem uma quantidade de veneno menor, com baixa toxicidade e ausência de indução de edema pulmonar, quando comparados com a mesma espécie em outras regiōes do país. $\mathrm{O}$ veneno apresenta ação neurotóxica, evidenciada pela presença de hiperatividade, taquipnéia, letargia, piloereção, sialorréia e congestão ocular. A baixa toxicidade do veneno, aliada à ausência de indução de edema pulmonar parecem ser os fatores principais que podem explicar a ausência de óbito pelo envenenamento por Tityus serrulatus registrados na Região Metropolitana do Salvador, Bahia.

\title{
Study of Tityus serrulatus (Scorpiones; Buthidae) venom proceeding from the state of Bahia, Brazil
}

\begin{abstract}
The scorpion Tityus serrulatus is known as the most important species according to the medical point of view, because it is what causes the most serious registered accidents for the Brazilian country. This work deals with the characterization of the poison of scorpion T. serrulatus in different regions of Bahia/Brazil, through the attainment of the $D L_{50}$ of the poison the determination of pulmonary edema induced for the poison and the characterization of the neurotoxic effects induced by the poison. The poison was obtained through electric stimulation using itself of a extractor (method of Brazil, 1949), developed specifically for this end. Male Swiss mice (18-22g) had been used to evaluate the toxicity for the method of Finney (1971). The pulmonary induction of edema was calculated by the difference of weight of the controlled and tested lung. The obtained DL50 was of $93.6 \mu \mathrm{g}$ mouse. We can attribute this high value to low toxicity of the poison for the animals of the studied region (Metropolitan Region of Salvador). The observed toxicity was of 3 to 7 times lower than that the poison of the same species in other regions of Brazil (São Paulo and Minas Gerais). Our results in the performed experiments to study acute lung edema had demonstrated that the poison of T. serrulatus of the State of the Bahia does not induce death.
\end{abstract}

Keywords: scorpion - venom; Tityus; T. serrulatus.

\section{REFERÊNCIAS}

AMARAL, C.F.S.; RESENDE, N.A.; FREIREMAIA, L. Acute pulmonary edema after Tityus serrulatus scorpion sting in children. Am. J. Cardiol., New York, v.71, p.242-245, 1993.

BARRIO, A.; VITAL BRAZIL, O. Verfahren der giftentnahme bei spinnen. Experientia, Basel, v.6, p.112-113, 1949.

BROWNELL, P.; POLIS, G. Scorpion biology and research. New York: Oxford University Press, 2001.

BÜCHERL, W. Venoms of tityinae. In BETTINI, S. Handbook of experimental pharmacology. New York: Springer Verlag, 1978. cap.14, p.371-378.

BÜCHERL, W.; BUCKLEY, E.E. Classification, biology and venom extraction of scorpions. In: BÜCHERL, W. (Ed.) Venomous animals and their venoms. New York: Academic Press, 1971. v.3, p.317-347.

CUPO, P. et al. Severe scorpion envenomation in Brazil: clinical, laboratory and anatomopathological aspects. R. Inst. Med. Trop. São Paulo, São Paulo, v.36, n.1, p.67-76, 1994. 
CUPO, P.; AZEVEDO-MARQUES, M.M.; HERING, S.E. Escorpionismo. In: CARDOSO, J.L.C. et al. Animais peçonhentos no Brasil: biologia, clínica e terapêutica dos acidentes. São Paulo: Sarvier, 2003. p.197-210.

FERNANDES, V.M.V. et al. Effects of ascorpion, toxin tityustoxinon the realese of $\left[{ }^{3} \mathrm{H}\right.$ ] dopamineof rat brain prefrontal cortical slices. Neurochem. Int., Oxford, v.44. 2004.

FINNEY, D. J. Probit analysis. $3^{\text {rd }}$.ed. London: Cambridge University Press, 1971.

FREIRE-MAIA, L. et al. Cholinergic and adrenergic effects of tityustoxin. Gen. Pharmacol., Exeter, v.7, p.115-121, 1976.

FREIRE-MAIA, L.; CAMPOS, J.A.; AMARAL, C.F.S. Approches to the treatment of scorpion envenoming. Toxicon, Oxford, v.32, n.9, p.1009-1014, 1994.

FREIRE-MAIA, L.; DINIZ, C.R. Pharmacological action of a purified scorpion toxin in the rat. Toxicon, Oxford, v.8, 1970.

FUKUTANI, K.F. et al. Atualização da escorpiofauna do Estado da Bahia, Brasil. In: CONGRESSO BRASILEIRO DE ZOOLOGIA, 25., 2004, Brasília. Resumos. Brasília, DF: Sociedade Brasileira de Zoologia, 2004. p.26.

HERING, S.E., AZEVEDO-MARQUES, M.M.; CUPO, P. Escorpionismo. In SCHWARTSMAN, S. Plantas venenosas e animais peçonhentos. São Paulo: Sarvier, 1992. cap.15, p.216-227.

KALAPOTHAKIS, E.; CHAVEZOLORTEGUI, C. Venom variability among several Tityus serrulatus specimens. Toxicon, Oxford, v.35, n.10, p.1523-1529, 1997.

LIRA-DA-SILVA, R.M. et al. Escorpionismo na Cidade do Salvador, Bahia, Brasil (19822000). R. Soc. Bras. Med. Trop., Rio de Janeiro, v.34, p.100, 2001. Supl.1.

LIRA-DA-SILVA, R.M. et al. Scorpions of medical importance in Bahia, Brazil (19822000). J.Venom. Anim.Toxins, Botucatu, v.3, n.1, p.249, 1997.
LIRA-DA-SILVA, R.M.; AMORIM, A.M.; BRAZIL, T.K. Envenenamento por Tityus stigmurus (Scorpiones; Buthidae) no Estado da Bahia, Brasil. R. Soc. Bras. Med. Trop., Rio de Janeiro, v.33, n.3, p.239-245, 2000.

LOURENÇO, W.R. et al. The evolution of scorpionism in Brazil in recent years. J. Venom Anim. Toxins., Botucatu, v.2, n.2, p.121-134, 1996.

LOURENÇO, W.R.; CLOUDSLEYTHOMPSON, J.L. Discovery of a sexual population of Tityus serrulatus, one of the morphs within the complex tityus stigmurus (Scorpiones; Buthidae). J. Arachnol., Lubbock, v.27, p.154-158, 1999.

LOURENÇO, W.R.; EICKSTEDT, V.R.D.von. Escorpiōes de importância médica. In: CARDOSO, J.L.C. et al. Animais peçonhentos no Brasil: biologia, clínica e terapêutica dos acidentes. São Paulo: Sarvier, 2003. p.182-197.

MAGALHĀES, M.M. et al. The mouse as an experimental model for Tityus serrulatus scorpion envenoming. Acta Cir. Bras., São Paulo, v.13, n.4, 1998.

MAGALHĀES, O.; TUPINAMBÁ, A. Escorpionismo. Brasil-Médico, Rio de Janeiro, n.29, p.28-29, 1938.

MANUAL de diagnóstico e tratamento de acidentes por animais peçonhentos. Brasília, DF: Fundação Nacional da Saúde, 1999.

MATOS, M. de et al. Lung oedema induced by Tityus serrulatus scorpion venom in the rat. Comp. Biochem. Physiol. C, Pharmacol.

Toxicol. Endocrinol., New York, v.118, n.2, p.143-148, 1997.

MAURANO, H.R. Do escorpionismo. 1915. Tese - Faculdade de Medicina do Rio de Janeiro, Rio de Janeiro, 1915.

NISHIKAWA, A.K. et al. Antigenic crossreactivity among the venoms from several species of Brazilian scorpions. Toxicon, Oxford, v.32, n.8, p.989-998, 1994.

RAHAV , G.; WEISS, A.T. Scorpion sting- 
induced pulmonary edema: scintigraphic evidence of cardiac dysfunction. Chest, Northbrook, v.97, p.1478-1480, 1990.

ROSENFELD, G. Acidentes por animais peçonhentos. In: VERONESI, R. (Ed.) Doenças infecciosas e parasitárias. Rio de Janeiro: Guanabara Koogan, 1976.

SCHWARTSMAN, S. Plantas venenosas e animais peçonhentos. São Paulo: Sarvier, 1992.
VIEIRA G.V. et al. Epidemiologia do acidente por escorpião na Grande São Paulo e municípios próximos, HVB, 1989/90. R. Soc. Bras. Med. Trop., Rio de Janeiro, v.29, p.238, 1996.

VITAL BRAZIL, O. Duração da atividade antitóxica dos soros (1916). Colet. Trab. Inst. Butantan., São Paulo, v.2, p.297-309, 1918.

Recebido em / Received: 03/02/2005 Aceito em / Accepted: 27/04/2005 